\title{
The sbvIMPROVER metagenomics diagnostics for inflammatory bowel disease challenge: results and lessons learned
}

\author{
Lusine Khachatryan \\ PMI R\&D, Philip Morris Products \\ S.A., Neuchâtel, Switzerland \\ Lusine.Khachatryan@pmi.com \\ Adrian Stan \\ PMI R\&D, Philip Morris Products \\ S.A., Neuchâtel, Switzerland \\ Adrian.Stan@pmi.com \\ Stephanie Boue \\ PMI R\&D, Philip Morris Products \\ S.A., Neuchâtel, Switzerland \\ Stephanie.Boue@pmi.com \\ Manuel C Peitsch \\ PMI R\&D, Philip Morris Products \\ S.A., Neuchâtel, Switzerland \\ Manuel.Peitsch@pmi.com
}

\author{
Carine Poussin \\ PMI R\&D, Philip Morris Products \\ S.A., Neuchâtel, Switzerland \\ Carine.Poussin@pmi.com \\ James Battey \\ PMI R\&D, Philip Morris Products \\ S.A., Neuchâtel, Switzerland \\ James.Battey@pmi.com \\ Nicolas Sierro \\ PMI R\&D, Philip Morris Products \\ S.A., Neuchâtel, Switzerland \\ Nicolas.Sierro@pmi.com \\ Julia Hoeng \\ PMI R\&D, Philip Morris Products \\ S.A., Neuchâtel, Switzerland \\ Julia.Hoeng@pmi.com
}

\author{
Yang Xiang \\ PMI R\&D, Philip Morris Products \\ S.A., Neuchâtel, Switzerland \\ Yang.Xiang@pmi.com \\ Giuseppe Lo Sasso \\ PMI R\&D, Philip Morris Products \\ S.A., Neuchâtel, Switzerland \\ Giuseppe.LoSasso@pmi.com \\ Nikolai V. Ivanov \\ PMI R\&D, Philip Morris Products \\ S.A., Neuchâtel, Switzerland \\ Nikolai.Ivanov@pmi.com
}

\begin{abstract}
A growing number of reports indicate the potential benefit of exploiting metagenomics for noninvasive IBD diagnostics. In order to in investigate the diagnostic potential of metagenomics data for IBD patients, we conducted the crowdsourced sbv IMPROVER Metagenomics Diagnosis for Inflammatory Bowel Disease Challenge.
\end{abstract}

Keywords-IBD, metagenomic, crowdsourcing

\section{Motivation and Aim}

Inflammatory bowel diseases (IBD) constitute a spectrum of chronic inflammatory disorders that affect the gastrointestinal tract. Ulcerative colitis (UC) and Crohn's disease (CD) are the two main clinically defined manifestations of IBD, each with distinctive clinical and pathological features. A growing number of reports showing changes in gut microbiota in subjects with IBD indicate the potential benefit of exploiting metagenomics for noninvasive IBD diagnostics.

\section{Methods}

In order to investigate the diagnostic potential of metagenomics data to discriminate IBD patients from non-
IBD subjects and, within the IBD group, to separate UC and CD subjects, we conducted the crowdsourced sbv IMPROVER Metagenomics Diagnosis for Inflammatory Bowel Disease Challenge, which was open to the worldwide scientific community from September 2019 to March 2020.

\section{Results}

Participants were provided either raw (sub-challenge 1) or processed (sub-challenge 2) independent train and test metagenomics data to develop and apply models for classifying metagenomics fecal samples from CD, UC, and non-IBD subjects. The challenge results will help answer a set of scientific questions, which include: whether metagenomics data are sufficiently informative for predicting IBD status with potential applications for IBD diagnostics; what is the nature of the most discriminative metagenomic features (taxonomy, pathway, or other), and are they distinct in UC and CD; and which predictive computational approach is the most accurate for each of the classification tasks?

In conclusion, the outcomes of the challenge will be summarized and shared with the scientific community to advance the research in the fields of IBD and diagnostics. 